\title{
AVALIAÇÃO DAS CARACTERÍSTICAS DE QUALIDADE DE NÉCTARES DE GOIABA LIGHT DE DIFERENTES MARCAS COMERCIALIZADAS EM LAVRAS/MG.
}

\section{EVALUATION OF QUALITY CHARACTERISTICS OF GUAVA NECTARS LIGHT OF DIFFERENT BRANDS MARKETED IN LAVRAS / MG.}

\author{
Larissa de Oliveira Ferreira Rocha ${ }^{1}$; Carlos José Pimenta ${ }^{2}$; Patrícia Aparecida Pimenta Pereira ${ }^{3}$ \\ ${ }^{1,2,3}$ Universidade Federal de Lavras - UFLA - Lavras - Brasil larioliv@ hotmail.com
}

\begin{abstract}
Resumo
O presente trabalho teve como objetivo estudar as características de qualidade de cinco marcas de néctar de goiaba "light" comercializados na cidade de Lavras/MG. As determinações de pH, acidez, sólidos solúveis $\left({ }^{\circ}\right.$ Brix), açúcares totais e cor foram realizadas conforme metodologia proposta. Avaliou-se a aceitação sensorial das amostras e a intenção de compra mediante equipe com 65 julgadores, utilizando escala hedônica estruturada. Os resultados das análises e do teste de aceitação foram avaliados por análise estatística univariada (ANOVA) e teste de médias (Tukey, $p \leq 0,05)$. Com base nas informações dos consumidores e nos resultados do teste afetivo, foram construídos histogramas. A análise multivariada qualitativa (análise de componentes principais) também foi empregada com o objetivo de compreender melhor a diferenciação das amostras analisadas. Em todos os atributos avaliados as amostras de néctar foram consideradas significativamente $(p \leq 0,05)$ diferentes. Observou-se que a marca B mostrou-se estatisticamente diferente das demais marcas, apresentando maior teor de sólidos solúveis, açúcares e acidez e menor valor de $\mathrm{pH}$. A amostra $C$ apresentou alto valor de $a^{*}$, indicando possuir cor vermelha mais intensa. Em relação a avaliação sensorial, observou-se que os consumidores apresentaram uma maior aceitação pela marca $B$ quando o sabor foi avaliado, e maior aceitação pela amostra $C$ quando a cor foi avaliada. A análise de componentes principais confirmou esses resultados $e$ permitiu concluir que houve uma maior tendência de aceitação por um néctar de goiaba "light" de cor avermelhada e de sabor adocicado e ácido, o que pode ser um parâmetro a ser seguido pelos fabricantes.
\end{abstract}

Palavras-chave: goiaba, avaliação físico-química e sensorial.

\section{Introdução}

A goiaba (Psidium guajava, L.) da família Myrtaceae é uma das frutas mais completas e equilibradas em relação ao valor nutritivo, destacando-se por ser uma excelente fonte de vitamina $\mathrm{C}$ (BRITO e BOLINI, 2008). Sua utilização destina-se a duas finalidades principais: consumo ao 
natural e industrialização. As frutas destinadas ao processamento industrial resultam na confecção de frutas em caldas ou na produção de polpa. A partir da polpa processada podem-se elaborar produtos como néctar, suco, doce em massa, geléia e geleiado, sorvete, ingredientes de produtos de laticínios, de panificação, entre outros (MARTIN e KATO, 1991).

Dados de 2004 indicam que a produção/consumo de sucos, néctares e drinques a base de frutas no Brasil foi de 350 milhões de litros, sendo que o de goiaba representou $6 \%$ de toda essa produção, apresentando uma tendência de crescimento, o que mostra a sua importância econômica no mercado nacional (KAWAGUTI et al., 2007). Isso se deve não somente ao seu alto conteúdo de ácido ascórbico e vitaminas, como também ao prazeroso e delicado sabor/aroma, que o suco de goiaba apresenta (CHAN, 1993). A recente descoberta de seu alto conteúdo em licopeno e suas características funcionais anticancerígenas tem induzido a um maior consumo da fruta (BRAMLEY, 2000).

Atualmente o mercado de sucos e néctares "lights" (bebidas com o conceito de menos calorias) é um mercado em expansão, devido principalmente às mudanças de hábitos alimentares e busca de qualidade de vida pelos consumidores. Outro fator foi a melhora do poder aquisitivo dos brasileiros (BRITO e BOLINI, 2009).

Segundo a Instrução Normativa $n^{\circ} 12$ (BRASIL, 2003) néctar de goiaba é a bebida não fermentada, obtida da dissolução, em água potável, da parte comestível da Goiaba (Psidium guajava, L.) e açúcares, destinado ao consumo direto, podendo ser adicionado de ácidos. E segundo a Instrução Normativa $n^{\circ} 30$ bebida de baixa caloria é a bebida não alcoólica e hipocalórica, devendo ter o conteúdo de açúcares adicionados normalmente na bebida convencional, inteiramente substituído por edulcorantes hipocalóricos e não calóricos naturais ou artificiais, exceto para o preparado sólido para refresco. A bebida de baixa caloria terá a designação da bebida convencional seguida do termo "de Baixa Caloria" e é permitido o uso do termo "light" no rótulo (BRASIL, 1999).

Os testes sensoriais de sucos de goiaba são de extrema importância, pois podem ser incluídos entre os de garantia de qualidade do produto, por ser uma medida multidimensional integrada capaz de detectar, de forma rápida, particularidades sensoriais que não podem ser detectadas por outros procedimentos analíticos e ainda ser capaz de avaliar se um produto é bem aceito ou não (MUÑOZ et al, 1992).

Entre os testes sensoriais disponíveis para se medir a aceitação e preferência dos consumidores com relação a um ou mais produtos, a escala hedônica, a escala de atitude e a do ideal são as mais utilizadas (MINIM, 2006). A escala hedônica estruturada de nove pontos é provavelmente o método afetivo mais utilizado devido à confiabilidade e validade de seus resultados, bem como sua simplicidade em ser utilizada pelos provadores (STONE E SIDEL, 1993). 
Os resultados de testes afetivos são, tradicionalmente, avaliados por análise de variância univariada e teste de médias (STONE e SIDEL, 1993). Outra forma de se avaliar os resultados da escala hedônica é a análise da distribuição de frequências dos valores hedônicos obtidos por cada amostra, através de histogramas. Os histogramas tornam possível a visualização da segmentação dos valores hedônicos de cada amostra, revelando o nível de aceitação e rejeição da mesma e permitindo a comparação dos desempenhos de duas ou mais amostras que participaram do estudo (BEHRENS et al., 1999).

Devido à mudança do mercado consumidor na busca por alimentos de menor aporte calórico e da ausência de trabalhos caracterizando esses produtos já inseridos no mercado, o presente trabalho teve como objetivo estudar as características físico-químicas e de cor de diferentes marcas de néctar de goiaba light comercializados na cidade de Lavras/MG, e também verificar a aceitação sensorial desses produtos, bem como avaliar quais as características que mais interferiram na sua aceitação.

\section{Material e Métodos}

\section{Amostragem}

Foram analisadas 5 marcas comerciais de néctares de goiaba light adquiridos no comércio local da cidade de Lavras, Minas Gerais. De cada marca foram adquiridos 2 litros de néctar em embalagem asséptica e dentro do prazo de validade. As marcas estudadas foram designadas de A, B, C, D, e E objetivando manter a idoneidade de seus fabricantes. Na Tabela 1 tem-se a composição especificada no rótulo de cada produto.

Tabela 1 - Ingredientes especificados no rótulo das diferentes marcas de néctares de goiaba light

\begin{tabular}{cl}
\hline Marcas & \multicolumn{1}{c}{ Ingredientes } \\
\hline $\mathrm{A}$ & $\begin{array}{l}\text { Água, polpa integral de goiaba, acidulante ácido cítrico, edulcorantes sucralose e acessulfame K e } \\
\text { aroma idêntico ao natural de goiaba. Não possui conservadores químicos. }\end{array}$ \\
B & $\begin{array}{l}\text { Água, edulcorantes: sucralose e acesulfame k, polpa de goiaba, aroma natural de goiaba, } \\
\text { acidulante: acido cítrico, vitamina C: ácido ascórbico. }\end{array}$ \\
$\mathrm{C}$ & $\begin{array}{l}\text { Água, polpa de goiaba, acidulante: ácido cítrico, edulcorantes: sucralose, acesulfame K, } \\
\text { antioxidante: ácido ascórbico. }\end{array}$ \\
Água potável, suco de goiaba; acidulante: ácido cítrico; antioxidante: ácido ascórbico; espessante: \\
goma gelana; edulcorantes artificiais: acesulfame k e sucralose; aromatizante: aroma idêntico ao \\
natural de goiaba e regulador de acidez citrato de sódio. \\
Água, polpa de goiaba, ácido ascórbico (vitamina C), acidulante ácido cítrico, aroma natural, \\
Edulcorantes sucralose e acesulfame k.
\end{tabular}

\section{Determinações físico-químicas}

As determinações físico-químicas dos néctares foram efetuadas no Laboratório Central do Departamento de Ciência dos Alimentos da Universidade Federal de Lavras. As análises foram 
realizadas em quadruplicata. A concentração de sólidos solúveis foi determinada através de leitura refratométrica a $20^{\circ} \mathrm{C}$, conforme Instituto Adolfo Lutz (IAL) (1985). O pH foi determinado utilizando-se o método eletroanalítico (potenciométrico) em peagâmetro Tecnal ${ }^{\mathrm{R}} \mathrm{Tec}^{3 \mathrm{MP}}$, segundo IAL (1985). A acidez total titulável foi determinada por titulação com $\mathrm{NaOH} 0,1$ molar e os resultados foram expressos em percentagem de ácido cítrico, segundo IAL (1985). Os açúcares foram determinados pelo método de Antrona, conforme Dische (1962).

\section{Avaliação da cor}

A cor dos néctares foi determinada em colorímetro molelo KONICA Minolta CR 400, sistema $L^{*} a^{*} b^{*}$, em que: $L^{*}$ : mede a luminosidade e varia de 100 (cem) para superfícies perfeitamente brancas até 0 (zero) para o preto; $\mathrm{a}^{*}$ : mede a intensidade de vermelho (+), e verde (-); b*: mede a intensidade de amarelo (+), e azul (-).

\section{Análise sensorial}

A aceitabilidade e a intenção de compra das amostras de néctar de goiaba light foram realizadas por 65 consumidores. Foram oferecidos aproximadamente $10 \mathrm{~mL}$ de cada amostra de néctar em copos descartáveis codificados com números de três dígitos. As amostras foram apresentadas de forma balanceada segundo Wakeling e McFie (1995). O teste foi realizado no período da tarde no Laboratório de Análise Sensorial da Universidade Federal de Lavras, empregando-se cabines individuais e luz branca.

Escalas hedônicas estruturadas de nove pontos, cujos extremos correspondem a desgostei extremamente (1) e gostei extremamente (9) foram utilizados no teste de aceitação dos néctares para a avaliação dos atributos cor e sabor, conforme Stone e Sidel (1993). Avaliou-se a intenção de compra do produto mediante escala estruturada de cinco pontos, cujos extremos correspondem a certamente não compraria (1) e certamente compraria (5), conforme Stone e Sidel (1993).

\section{Análise estatística}

Os resultados das análises e do teste de aceitação foram avaliados por análise estatística univariada (ANOVA) e teste de médias (Tukey, $\mathrm{p} \leq 0,05$ ), utilizando o software Sisvar (FERREIRA, 2000). Com base nas informações dos consumidores e nos resultados do teste afetivo, foram construídos histogramas.

A análise multivariada qualitativa (análise de componentes principais) também foi empregada com o objetivo de compreender melhor a diferenciação das amostras analisadas. A análise de componentes principais permite uma análise global dos resultados e sugere quais atributos ou 
descritores mais caracterizam as amostras. O gráfico foi gerado empregando-se a Análise de Componentes Principais utilizando-se o programa Matlab, versão 7.8.0.347 (2009).

\section{Resultados e Discussão}

\section{Determinações físico-químicas}

A Tabela 2 mostra o resultado das análises químicas e físico-químicas dos néctares de goiaba light de diferentes marcas comerciais. Observou-se que em todos os parâmetros analisados houve diferença significativa $(\mathrm{p} \leq 0,05)$ entre as amostras de néctar (Tabela 2$)$.

Tabela 2 - Características químicas e físico-químicas de 5 diferentes marcas de néctares de goiaba light comercializados na cidade de lavras-minas gerais *

\begin{tabular}{clllll}
\hline \multicolumn{1}{c}{ Análises } & \multicolumn{5}{c}{ Marcas } \\
\cline { 2 - 6 } & \multicolumn{1}{c}{$\mathbf{A}$} & \multicolumn{1}{c}{$\mathbf{B}$} & \multicolumn{1}{c}{$\mathbf{C}$} & \multicolumn{1}{c}{ D } & \multicolumn{1}{c}{$\mathbf{E}$} \\
\cline { 2 - 6 } Sólidos solúveis $\left({ }^{\circ}\right.$ Brix) & $2,80 \mathrm{~d} \pm 0,08$ & $6,55 \mathrm{a} \pm 0,06$ & $2,90 \mathrm{~cd} \pm 0$ & $3,03 \mathrm{~b} \pm 0,05$ & $2,95 \mathrm{bc} \pm 0,06$ \\
$\mathrm{pH}$ & $3,91 \mathrm{a} \pm 0$ & $3,52 \mathrm{e} \pm 0$ & $3,76 \mathrm{~b} \pm 0,04$ & $3,58 \mathrm{~d} \pm 0,01$ & $3,71 \mathrm{c} \pm 0,0$ \\
Acidez total em ácido cítrico $(\mathrm{g} / 100 \mathrm{~g})$ & $0,35 \mathrm{~b} \pm 0,04$ & $0,48 \mathrm{a} \pm 0,32$ & $0,37 \mathrm{~b} \pm 0,03$ & $0,38 \mathrm{~b} \pm 0,0$ & $0,38 \mathrm{~b} \pm 0,0$ \\
Açúcares totais $(\mathrm{g} / 100 \mathrm{~g})$ & $2,40 \mathrm{c} \pm 0,18$ & $5,93 \mathrm{a} \pm 0,14$ & $2,65 \mathrm{c} \pm 0,02$ & $2,59 \mathrm{c} \pm 0,02$ & $2,99 \mathrm{~b} \pm 0,09$ \\
\hline
\end{tabular}

* Médias nas linhas seguidas por letras iguais não diferem entre si, a 5\% de significância, pelo teste de Tukey. Valores médios (média \pm desvio padrão) de quadruplicatas.

Fonte: Autores

Na Tabela 2, observa-se que não houve grandes variações entre as marcas em relação à acidez, todas foram consideradas estatisticamente semelhantes ao nível de 5\% de probabilidade exceto a marca B que apresentou a maior acidez.

$\mathrm{O}$ pH é de suma importância para a formulação das bebidas, uma vez que nunca deve ser superior a 4,5, visto que acima deste valor pode favorecer o crescimento do Clostridium botulinum. Entretanto, na tabela 2 pode-se observar que todas as amostras apresentam pH abaixo de 4,5. No entanto, a marca A apresentou um pH de 3,91, ligeiramente mais elevado que as demais.

Os sólidos solúveis totais dos sucos compreendem fundamentalmente, os açúcares e os ácidos orgânicos (YÚFERA, 1997). A marca B apresentou um teor de sólidos solúveis estatisticamente superior aos demais, esse maior teor pode ter ocorrido em função da matéria-prima utilizada ou por adição de açúcar o que não é permitido.

Os açúcares totais variaram entre 2,40 e 5,93, sendo que, novamente a marca B apresentou valor estatisticamente superior aos demais em relação ao teor de açúcar, e como foi mencionado anteriormente esse fato pode ter ocorrido em função da matéria-prima utilizada ou por adição de açúcar.

Godoy et al. (1998) estudando a estabilização de néctares de goiaba, encontrou menores valores de acidez $(0,157-0,190)$ e maiores valores de $\mathrm{pH}(3,91-3,99)$. 


\section{Avaliação de cor}

Em relação à cor, observou-se que em todos os parâmetros analisados houve diferença significativa $(\mathrm{p} \leq 0,05)$ entre as amostras de néctar (Tabela 3$)$.

Tabela 3 - Médias dos componentes da cor $\left(\mathrm{L}^{*}, \mathrm{a}^{*}\right.$ e $\left.\mathrm{b}^{*}\right)$ para as cinco amostras néctares de goiaba light *

\begin{tabular}{cccc}
\hline AMOSTRA & $\mathbf{L}^{*}$ & $\mathbf{a}^{*}$ & $\mathbf{b}^{*}$ \\
\hline $\mathbf{A}$ & $34,44 \mathrm{a} \pm 0,08$ & $6,81 \mathrm{c} \pm 0,07$ & $11,59 \mathrm{a} \pm 0,08$ \\
B & $28,83 \mathrm{~d} \pm 0,11$ & $5,13 \mathrm{e} \pm 0,08$ & $8,73 \mathrm{c} \pm 0,10$ \\
C & $31,40 \mathrm{c} \pm 0,24$ & $9,39 \mathrm{a} \pm 0,05$ & $9,45 \mathrm{~b} \pm 0,11$ \\
$\mathbf{D}$ & $34,85 \mathrm{a} \pm 0,15$ & $5,65 \mathrm{~d} \pm 0,06$ & $5,95 \mathrm{~d} \pm 0,08$ \\
E & $32,85 \mathrm{~b} \pm 0,58$ & $7,56 \mathrm{~b} \pm 0,20$ & $8,31 \mathrm{c} \pm 0,39$ \\
\hline
\end{tabular}

* Médias nas colunas seguidas por letras iguais não diferem entre si, a 5\% de significância, pelo teste de Tukey. Valores médios (média \pm desvio padrão) de quadruplicatas.

Fonte: Autores

O valor $\mathrm{L}^{*}$ expressa a luminosidade ou claridade da amostra, quanto mais próximo de 100 , mais clara é a amostra. Já valores de a* positivos indicam tendência a coloração vermelha e valores de $b^{*}$ positivos expressam maior intensidade de amarelo.

Analisando os valores $\mathrm{a}^{*} \mathrm{e} \mathrm{b}^{*}$ é possível afirmar que as amostras de néctar de goiaba tenderam às cores vermelho e amarela e quanto ao valor $\mathrm{L}^{*}$ apresentaram claridade intermediária tendendo a baixa claridade (Tabela 3). Resultados semelhantes foram encontrados por Kawaguti et al. (2007) quando avaliou a cor de néctares de goiaba industrializados.

As marcas A e D foram consideradas estatisticamente semelhantes, em relação ao parâmetro L*, apresentando uma coloração mais clara que as demais. Em relação ao parâmetro a*, todas as amostras foram consideradas estatisticamente diferentes $(\mathrm{p} \leq 0,05)$, a amostra $\mathrm{C}$ apresentou o maior valor de $\mathrm{a}^{*}$, indicando possuir cor vermelha mais intensa. As amostras B e E foram consideradas estatisticamente semelhantes em relação ao parâmetro b*.

\section{Análise sensorial}

As médias de aceitação para cor e sabor dos néctares encontram-se na Tabela 4.

Tabela 4 - Médias dos atributos de cor e sabor para cada amostra de néctar de goiaba light *

\begin{tabular}{ccc}
\hline AMOSTRA & COR & SABOR \\
\hline A & $6,19 \mathrm{bc}$ & $5,59 \mathrm{ab}$ \\
B & $6,45 \mathrm{ab}$ & $6,05 \mathrm{a}$ \\
C & $7,26 \mathrm{a}$ & $4,64 \mathrm{~b}$ \\
D & $5,45 \mathrm{c}$ & $5,07 \mathrm{ab}$ \\
E & $6,40 \mathrm{abc}$ & $5,07 \mathrm{ab}$ \\
\hline DMS $^{*}$ & 0,97 & 1,21
\end{tabular}

* Médias nas colunas seguidas por letras iguais não diferem entre si, a 5\% de significância, pelo teste de Tukey. * DMS = diferença mínima significativa.

Fonte: Autores 
A análise de variância, entre as amostras, mostrou haver diferença significativa $(p \leq 0,05)$ na aceitação das amostras em relação à cor e sabor dos néctares.

Em relação à cor, a amostra $\mathrm{C}$ apresentou à maior nota $(7,26)$ e a amostra $\mathrm{D}$ a menor $(5,45)$. As amostras B e C cujas médias de aceitação foram 6,05 ("gostei ligeiramente") e 4,64 ("indiferente"), respectivamente, apresentaram diferença significativa entre si, em relação ao sabor.

A Figura 1 mostra os histogramas de distribuição das notas das amostras. Analisando-se o histograma das notas para a cor, pode-se verificar que a amostra $\mathrm{C}(66,7 \%)$ apresentou a maior porcentagem de notas entre 8 ("gostei muito) e 9 ("gostei extremamente"), já a amostra D obteve a maior porcentagem de julgamentos $(47,62 \%)$ na região de rejeição do produto (notas menores que 5). Em relação ao atributo sabor, a amostra B (50\%) apresentou a maior porcentagem de notas entre 7 ("gostei moderadamente") e 9 ("gostei extremamente"), indicando maior aceitação dessa amostra, e a amostra D a menor aceitação, já que obteve $57,14 \%$ dos julgamentos na região de rejeição.

Figura 1 - Histograma de frequiência das notas atribuídas às amostras em relação aos atributos sabor e cor.

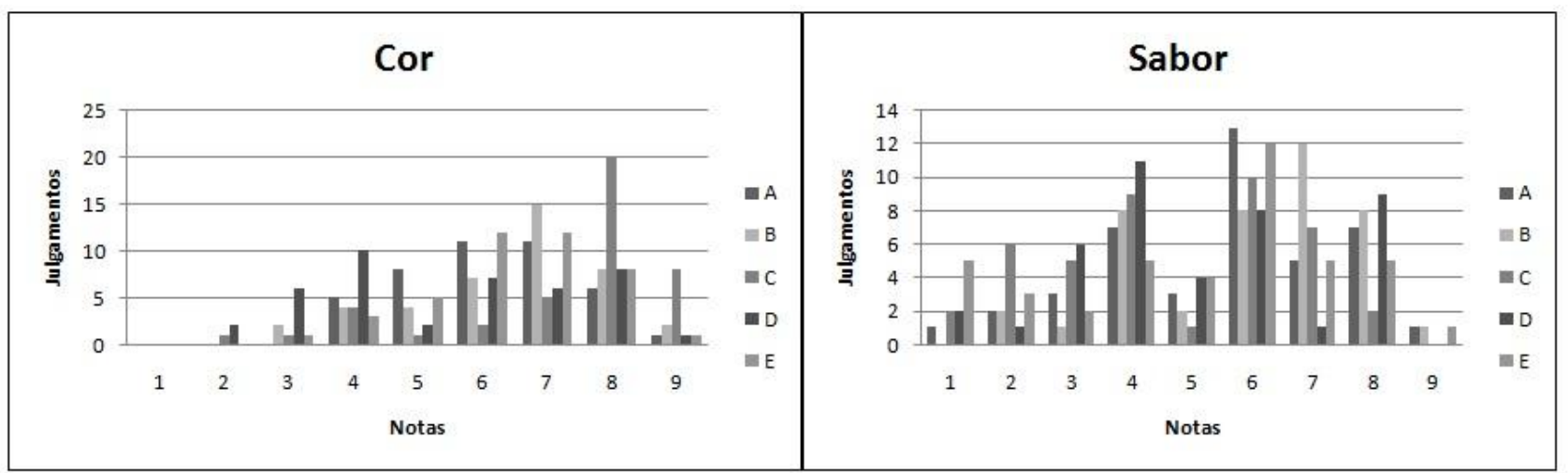

Fonte: Autores

Observou-se que os consumidores apresentaram uma maior aceitação pela marca B quando o sabor foi avaliado. A amostra $\mathrm{C}$ apresentou maior aceitação quando a cor foi avaliada, já a amostra D foi considerada a menos aceita nos dois atributos avaliados.

A Figura 2 comprova os resultados obtidos nos histogramas de freqüência para cor e sabor dos produtos avaliados.

A amostra D apresentou o maior índice de rejeição já que 55\% dos provadores certamente não comprariam ou possivelmente não comprariam esse produto. Já a amostra B apresentou o maior índice de aprovação, já que 52\% dos provadores possivelmente comprariam ou certamente comprariam o produto, indicando que o atributo sabor foi o que mais influenciou os provadores com relação à intenção de compra. 
Figura 2 - Histograma de frequiência das notas atribuídas às amostras em relação à intenção de compra.

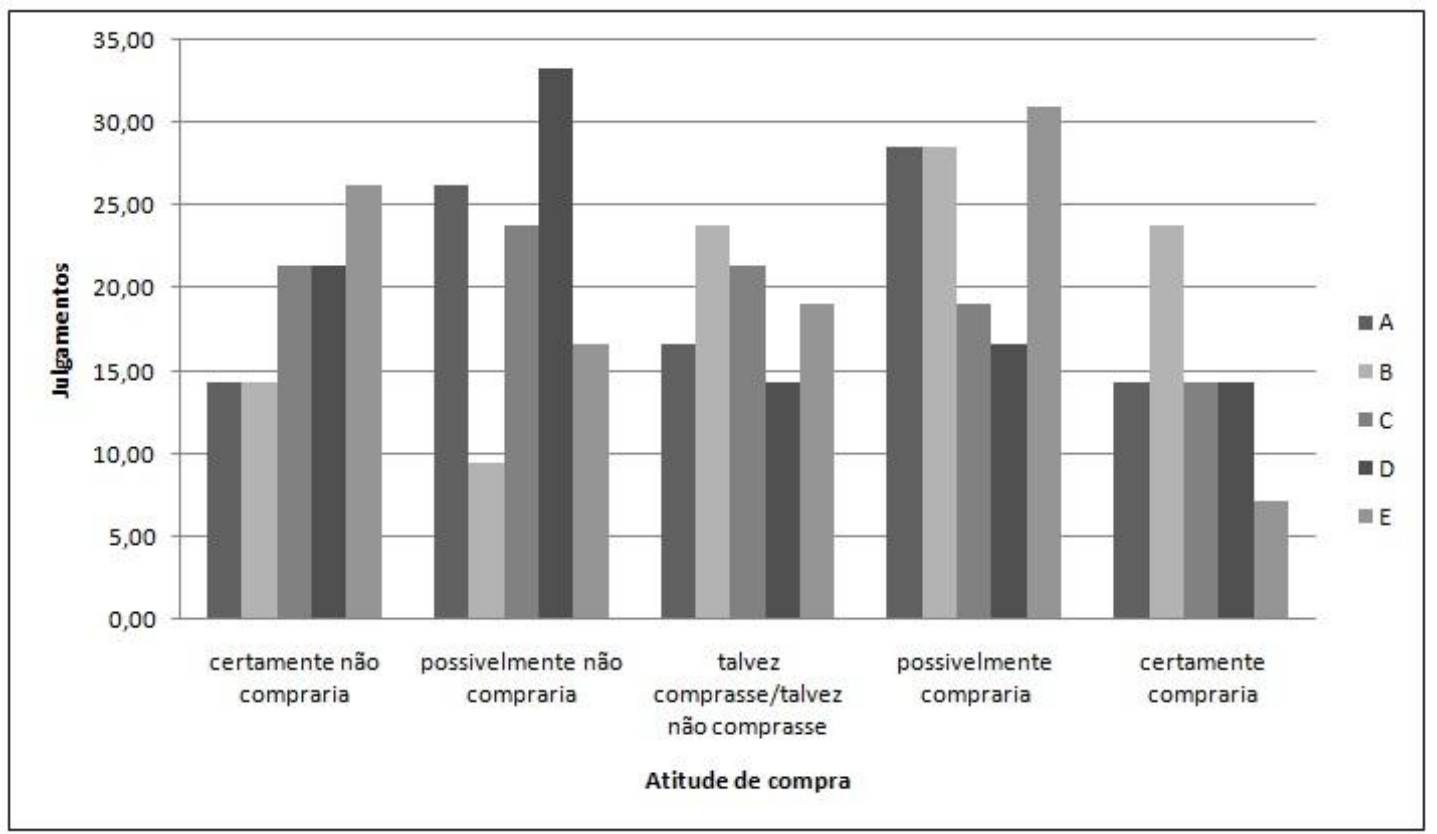

Fonte: Autores

Com o objetivo de compreender melhor a diferenciação das amostras analisadas, fez-se um estudo multivariado dos dados. A análise de componentes principais foi escolhida por permitir uma discussão dos resultados quando explorados considerando-se o peso de todas as medidas obtidas experimentalmente e sensorialmente. Na Figura 3 são mostrados os resultados gráficos que revelam a separação das amostras e as variáveis mais importantes nessa separação.

Figura 3 - Análise dos componentes principais.

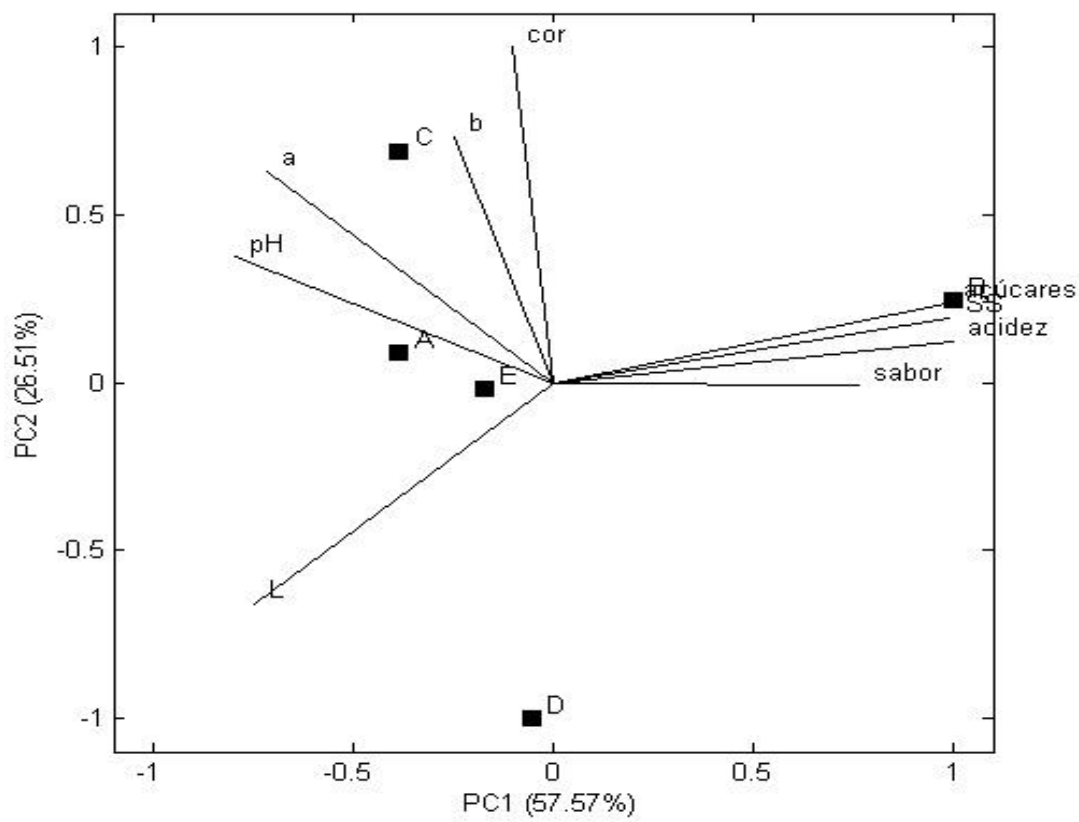

Fonte: Autores 
O primeiro componente principal (PC1) explicou 57,57\% e o segundo (PC2) explicou 26,51\% da variabilidade das respostas.

$\mathrm{Na}$ análise de componentes principais (ACP), os descritores são representados como vetores, sendo que, os que se apresentam longos, ao serem decompostos em um eixo PC, explicam mais a variabilidade entre as amostras mostradas naquele CP (DUTCOSKY, 2007). Verifica-se na Figura 3 que os $57,57 \%$ da variabilidade entre as amostras, explicada pelo PC1, foram devidos, principalmente, aos descritores: açúcar, sólidos solúveis e acidez. A variabilidade no PC2 (26,51\%) está associada à cor.

Vetores próximos uns dos outros indicam descritores que possivelmente apresentaram alta correlação entre si. Assim analisando a Figura 3, é possível sugerir correlação positiva entre os descritores açúcar, sólidos solúveis, acidez e sabor.

$\mathrm{Na}$ análise de componentes principais, as amostras localizam-se na mesma região dos vetores (descritores) que as caracterizam. Portanto, através do gráfico (Figura 3), pode-se observar os parâmetros instrumentais de cor $\left(\mathrm{a}^{*} \mathrm{e} \mathrm{b}^{*}\right)$ e o atributo sensorial de cor caracterizando a amostra $\mathrm{C}$, bem como o atributo sabor e os parâmetros físico-químicos (sólidos solúveis, acidez e açúcares) caracterizando a amostra B. Esses resultados encontram-se de acordo com os obtidos na avaliação físico-química e sensorial, visto que, a amostra B apresentou os maiores valores de sólidos solúveis, acidez e açúcares na avaliação físico-química e foi a amostra mais aceita no teste sensorial em relação ao sabor, já a amostra $\mathrm{C}$ apresentou-se diferente das demais marcas na análise instrumental de cor e maior aceitação no atributo de cor avaliado.

\section{Conclusão}

Em todos os atributos avaliados as amostras de néctar de goiaba light foram consideradas significativamente $(\mathrm{p} \leq 0,05)$ diferentes. Observou-se que a marca B mostrou-se estatisticamente diferente das demais marcas em todos os atributos avaliados, apresentando maior teor de sólidos solúveis, açúcares e acidez e menor valor de $\mathrm{pH}$.

Analisando os valores $a^{*}$ e $b^{*}$ é possível afirmar que as amostras de néctar de goiaba tenderam às cores vermelho e amarela e quanto ao valor $\mathrm{L}^{*}$ apresentaram claridade intermediária tendendo a baixa claridade. A amostra $\mathrm{C}$ apresentou alto valor de $\mathrm{a}^{*}$, indicando possuir cor vermelha mais intensa que as demais.

Em relação à avaliação sensorial, observou-se que os consumidores apresentaram uma maior aceitação pela marca B quando o sabor foi avaliado, e maior aceitação pela amostra $\mathrm{C}$ quando a cor foi avaliada. A análise de componentes principais confirmou esses resultados e permitiu concluir 
que houve maior tendência de aceitação por um néctar de goiaba light de cor avermelhada e de sabor adocicado e ácido, o que pode ser um parâmetro a ser seguido pelos fabricantes.

\begin{abstract}
This work aimed to study the quality of five brands of guava nectar light marketed in Lavras / MG. Measurements of $\mathrm{pH}$, acidity, soluble solids $\left({ }^{\circ}\right.$ Brix), total sugars and color were performed according to the proposed methodology. We evaluated the sensory acceptance of samples and purchase intent by the team with 65 judges using hedonic scale. The results of the analysis and the acceptance test were evaluated by univariate statistical analysis (ANOVA) and mean test (Tukey, $p$ $\leq$ 0.05). Based on information from consumers and affective test results, histograms were constructed. Multivariate Qualitative (principal component analysis) was also employed in order to better understand the differentiation of the samples. In all the attributes evaluated samples of nectar were found significantly ( $p \leq 0.05$ ) different. It was observed that the brand $B$ was statistically different from the other brands, with higher soluble solids, sugars and acidity and lower $p H$ value. The sample had a high value of $C a^{*}$ indicating red have more intense. For sensory evaluation, we found that consumers had a greater acceptance by the brand $B$ when the flavor was evaluated and the greater acceptance when the color sample $C$ was evaluated. The principal component analysis confirmed these results and concluded that there was a greater tendency to acceptance by a guava juice reddish color and sweet flavor and acid, which can be a parameter to be followed by manufacturers.
\end{abstract}

Key-words: guava; physic-chemical evaluation; sensory evaluation.

\title{
Referências
}

BEHRENS, J. H.; SILVA, M. A. A. P.; WAKELING, I. N. Avaliação da aceitação de vinhos brancos varietais brasileiros através de testes sensoriais afetivos e técnica multivariada de mapa de preferência interno. Ciência e Tecnologia de Alimentos, v. 19, n. 2, p.214-220 , 1999. http://dx.doi.org/10.1590/S0101-20611999000200011

BRAMLEY, P.M. Is lycopene beneficial to human health? Phytochemistry, v. 54, n. 3, p. 233-236, 2000. http://dx.doi.org/10.1016/S0031-9422(00)00103-5

BRASIL. Ministério da Agricultura, Pecuária e Abastecimento. Instrução normativa n ${ }^{\circ} \mathbf{1 2}$, de 04 de setembro de 2003. Aprova o regulamento técnico geral para fixação dos padrões de identidade e qualidade para suco tropical, disponível em: 〈http://extranet.agricultura.gov.br〉. Acesso em 11 mar. 2008.

BRASIL. Ministério da Agricultura. Secretaria da Defesa Agropecuária. Instrução normativa DAS n ${ }^{\circ} 30$, de 27 de setembro de 1999. Regulamento técnico geral para fixação dos padrões de identidade e qualidade para bebida dietética e de baixa caloria, disponível em: 〈http://extranet.agricultura.gov.br>. Acesso em 11 mar. 2008.

BRITO, C. A. K.; BOLINI, H. M. A. Análise da aceitação de néctares de goiaba por testes afetivos e mapa de preferência interno. Revista Brasileira de Tecnologia Agroindustrial, v. 02, n. 01, p. 67-80, 2008. http://dx.doi.org/10.3895/S1981-36862008000100006

BRITO, C. A. K.; BOLINI, H. M. A. Perfil sensorial de edulcorantes em néctar de goiaba. Alimentos e Nutrição, v. 20, n. 4, p. 561-572, out./dez., 2009.

CHAN, Jr., H. T. Passion fruit, papaya, and guava juices. In: NAGY, S.; CHEN, C.S.; SHAW, P.E. (Ed). Fruit Juice Processing Technology. Auburndale: Agscience, 1993. p. 335-349.

DISCHE, Z. General Color reactions. In: WHISTLER, R. L.; WOLFRAM, M. L. (Ed.).Carbohydrate chemistry. New York: Academic, 1962. p. 477-512.

DUTCOSKY, S. D. Análise sensorial de alimentos. 2. ed., Curitiba: Champagnat, 2007. 239 p.

FERREIRA, D. F. Análise estatística por meio do SISVAR para Windows, versão 4.0. In: Reunião Anual da Região Brasileira da Sociedade Internacional de Biometria, 45., 2000, São Carlos. Anais... 
GODOY, R. C. B.; ANTUNES, P. L.; ZONTA, E. P. Estabilização, de néctar de goiaba (Psidium Guayava L.) Com gomas xantana, carragena e amido ceroso. Revista Brasileira de Agrociência, v. 2, n. 2, p. 105-110, 1998.

INSTITUTO ADOLFO LUTZ. Normas Analíticas do Instituto Adolfo Lutz: métodos químicos e físicos para análise de alimentos. 3. ed. São Paulo, 1985. 533 p.

KAWAGUTI, H. Y.; CIAMPONE, S.; BARROS-MARCELlINI, A. M.; HOFSETZ, K.; PEREZ, R. M.; CAVAlHEIRO, S. F. L.; SIQUEIRA, P. B.; PIO, T. F.; BRITO, A. K. B.; BOLINI, H. M. A. Perfil sensorial e aceitação de néctares de goiaba industrializados. Revista Brasileira de Tecnologia Agroindustrial, v. 01, n. 02, p. 7082, 2007. http://dx.doi.org/10.3895/S1981-36862007000200008

MARTIN, Z. J.; KATO, K. Matéria-prima. In: Instituto de Tecnologia de Alimentos. Goiaba: cultura, material-prima, processamento e aspectos econômicos. 2 ed. Campinas, 1991, p. 141-175 (ITAL, Série frutas tropicais, 6)

MINIM, V.P.R. Análise sensorial: estudos com consumidores. Viçosa, MG: ed. UFV, 2006. 225 p.

MUÑOZ, A. M.; CIVILle, G. V.; CARR, B. T. Sensory evaluation in quality control. New York: Van Nostrand Reinhold, 1992. 240 p.

STONE, H. S.; SIDEL, J. L. Sensory Evaluation Practices, Academic Press, San Diego, CA, 1993, 308p.

WAKELING, I. N.; MAC FIE, H. J. H. Designing consumer trials balanced for first And higher orders of carry-over effect when only a subset of $k$ samples from $t$ may be tested. Food Quality and Preference, v. 6, n. 4, p. 299-308, 1995.

YÚFERA, E. P. Química de los alimentos. Madrid: Sintesis,1997. 459 p.

Submetido em 04 out. 2011, Aceito para publicação em 21 dez. 2012. 